\title{
The Brauer-Manin obstruction for curves having split Jacobians
}

\author{
par SAMIR SIKSEK
}

\begin{abstract}
RÉSUMÉ. Soit $X \rightarrow \mathcal{A}$ un morphisme (qui n'est pas constant) d'une courbe $X$ vers une variété abélienne $\mathcal{A}$, tous définis sur un corps de nombres $k$. Supposons que $X$ ne satisfait pas le principe de Hasse. Nous donnons des conditions suffisantes pour que l'obstruction de Brauer-Manin soit la seule obstruction au principe de Hasse. Ces conditions suffisantes sont légèrement plus fortes que de supposer que $\mathcal{A}(k)$ et $\amalg(\mathcal{A} / k)$ sont finis.
\end{abstract}

Abstract. Let $X \rightarrow \mathcal{A}$ be a non-constant morphism from a curve $X$ to an abelian variety $\mathcal{A}$, all defined over a number field $k$. Suppose that $X$ is a counterexample to the Hasse principle. We give sufficient conditions for the failure of the Hasse principle on $X$ to be accounted for by the Brauer-Manin obstruction. These sufficiency conditions are slightly stronger than assuming that $\mathcal{A}(k)$ and $\amalg(\mathcal{A} / k)$ are finite.

\section{Introduction}

Let $k$ be a number field, and let $\mathbb{A}_{k}$ be the adèles of $k$. Suppose that $X$ is a smooth and projective variety over $k$. We say that $X$ is a counterexample to the Hasse principle (or that the Hasse principle fails for $X$ ) if $X$ has no $k$-rational points but has rational points over all the completions of $k$. More concisely, $X$ is a counterexample to the Hasse principle if $X\left(\mathbb{A}_{k}\right) \neq \emptyset$ but $X(k)=\emptyset$.

Suppose that $X\left(\mathbb{A}_{k}\right) \neq \emptyset$. The global reciprocity applied to the BrauerGrothendieck group $\operatorname{Br}(X)$ defines a certain subset $X\left(\mathbb{A}_{k}\right)^{\operatorname{Br}(X)} \subseteq X\left(\mathbb{A}_{k}\right)$ that contains the diagonal image of $X(k)$. Hence if $X\left(\mathbb{A}_{k}\right)^{\operatorname{Br}(X)}=\emptyset$, it is clear that the Hasse principle fails for $X$. In this case one says that the failure of the Hasse principle for $X$ is accounted for by the Brauer-Manin obstruction.

Now let $X$ be a smooth projective curve over a number field $k$, let $\mathcal{J}_{X}$ be its Jacobian, and $\amalg\left(\mathcal{J}_{X} / k\right)$ be the Tate-Shafarevich group of $\mathcal{J}_{X} / k$. It is an open question whether or not all counterexamples to the Hasse principle on curves can be accounted for by the Brauer-Manin obstruction.

Manuscrit reçu le 6 juin 2003. 
(The answer to the same question for surfaces is known to be negative, see [7, Chapter 8]). We know of conditional answers in the following two situations:

(1) If $X$ has no $k$-rational divisor class of degree 1 then the (conjectured) finiteness of $\amalg\left(\mathcal{J}_{X} / k\right)$ implies that $X\left(\mathbb{A}_{k}\right)^{\operatorname{Br}(X)}=\emptyset$; see [7, Cor. 6.2.5].

(2) If $X(k)=\emptyset$ and $\mathcal{J}_{X}(k)$ is finite then again the (conjectured) finiteness of $\amalg\left(\mathcal{J}_{X} / k\right)$ implies that $X\left(\mathbb{A}_{k}\right)^{\operatorname{Br}(X)}=\emptyset$; this theorem is due to Scharaschkin, see [6] or [7, Cor. 6.2.5].

Scharaschkin's theorem is proved using an embedding $X \hookrightarrow \mathcal{J}_{X}$ corresponding to a $k$-rational divisor of degree 1 . If the Jacobian $\mathcal{J}_{X}$ splits over $k$, then for every factor $\mathcal{A}$ we have a non-constant morphism $\phi: X \rightarrow \mathcal{A}$. In this case it is natural to ask whether results similar to Scharaschkin's theorem can be obtained, with the finiteness of $\mathcal{J}_{X}(k)$ and $\amalg\left(\mathcal{J}_{X} / k\right)$ replaced by the weaker hypotheses: $\mathcal{A}(k)$ and $\amalg(\mathcal{A} / k)$ are finite. It is the purpose of this paper to show that this can be done under one extra assumption.

To state our main theorem we need to set up some notation. Suppose as above that $\phi: X \rightarrow \mathcal{A}$ is a non-constant morphism from a smooth projective curve $X$ to an abelian variety $\mathcal{A}$, all defined over a number field $k$. It is straightforward to see that $\phi(X)$ must be a complete (and irreducible) curve on $\mathcal{A}$, and that the induced map $X \rightarrow \phi(X)$ is therefore a finite morphism. We define the degree of $\phi$, denoted $\operatorname{deg}(\phi)$, to be the degree of this finite morphism $X \rightarrow \phi(X)$. If $Q \in \mathcal{A}$ such that $\phi^{-1}(Q) \neq \emptyset$, then let $l_{Q} / k$ be the compositum of the residue fields of all points in $\phi^{-1}(Q)$. Clearly $l_{Q} / k$ is a Galois extension.

We are now ready to state our two theorems.

Theorem 1.1. Let $k$ be a number field, and let $X$ be a smooth projective curve defined over $k$. Suppose $\phi: X \rightarrow \mathcal{A}$ is a non-constant morphism from $X$ to an abelian variety $\mathcal{A}$ (with $\phi$ and $\mathcal{A}$ also defined over $k$ ), such that $\mathcal{A}(k)$ and $\amalg(\mathcal{A} / k)$ are both finite. Suppose also that for all of (the finitely many) $Q \in \mathcal{A}(k)$ the following condition is satisfied: either $\phi^{-1}(Q)$ is empty, or else there exists an element of $\mathrm{Gal}\left(l_{Q} / k\right)$ that acts freely on $\phi^{-1}(Q)$. Then $X(k)=X\left(\mathbb{A}_{k}\right)^{\operatorname{Br}(X)}=\emptyset$.

Theorem 1.2. Let $k$ be a number field, and let $X$ be a smooth projective curve defined over $k$ such that $X(k)=\emptyset$. Suppose $\phi: X \rightarrow \mathcal{A}$ is a nonconstant morphism from $X$ to an abelian variety $\mathcal{A}$ (with $\phi$ and $\mathcal{A}$ also defined over $k)$. If $\mathcal{A}(k)$ and $\amalg(\mathcal{A} / k)$ are both finite, and if $\operatorname{deg}(\phi)<6$ then $X\left(\mathbb{A}_{k}\right)^{\operatorname{Br}(X)}=\emptyset$.

We will delay the proofs of our theorems till Section 3. First we give an example, showing that our results answer an interesting question asked by Coray and Manoil. 


\section{An Example}

Let $X / \mathbb{Q}$ be the genus 2 curve defined by

$$
X: \quad s^{2}=2\left(t^{3}+7\right)\left(t^{3}-7\right) .
$$

In [3, page 183] Coray and Manoil showed that the curve $X$ is a counterexample to the Hasse principle, despite possessing a rational divisor of degree 1. Coray and Manoil ask if this counterexample can be accounted for by the Brauer-Manin obstruction; we answer their question positively.

It is convenient to recount the proof that $X(\mathbb{Q})=\emptyset$. Let $E_{1}$ be the elliptic curve

$$
E_{1}: \quad y^{2}=x^{3}-392,
$$

and note that there is a morphism $X \rightarrow E_{1}$ given by $(t, s) \mapsto\left(2 t^{2}, 2 s\right)=$ $(x, y)$. It is noted in [3] that $E_{1}$ has only one rational point at infinity. Since the points on $X$ above the point at infinity on $E_{1}$ are not rational, we deduce that $X(\mathbb{Q})=\emptyset$.

Using a PARI/GP program of Tom Womack ${ }^{1}$ we find that the curve $E_{1}$ has analytic rank 0. It follows from a well-known theorem of Kolyvagin [5] that the Tate-Shafarevich group of $E_{1}$ is finite. Moreover, the map $\phi$ has degree 2. We deduce by Theorem 1.2 that $X\left(\mathbb{A}_{\mathbb{Q}}\right)^{\operatorname{Br}(X)}=\emptyset$ and so this counterexample is (unconditionally) accounted for by the Brauer-Manin obstruction.

We note in passing that the theorem of Scharaschkin cited in the introduction does not apply to $X$ since $\mathcal{J}_{X}(\mathbb{Q})$ is infinite. To see this, note that $\mathcal{J}_{X}$ is isogenous (see [1, page 155]) to $E_{1} \times E_{2}$ where

$$
E_{2}: \quad y^{2}=x^{3}+19208 \text {. }
$$

Moreover, using Cremona's program ${ }^{2}$ mwrank [4], we find that $E_{2}(\mathbb{Q})$ has rank 1. This shows that $\mathcal{J}_{X}(\mathbb{Q})$ is infinite.

\section{Proofs of the Theorems}

Proof of Theorem 1.1. We want to show that $X\left(\mathbb{A}_{k}\right)^{\operatorname{Br}(X)}=\emptyset$. Thus suppose $\left(P_{v}\right)_{v} \in X\left(\mathbb{A}_{k}\right)^{\operatorname{Br}(X)}$ and we would like to deduce a contradiction.

From the functorial properties of the Brauer-Manin pairing [7, page 102] we know that $\left(\phi\left(P_{v}\right)\right)_{v}$ is in $\mathcal{A}\left(\mathbb{A}_{k}\right)^{\operatorname{Br}(\mathcal{A})}$. The finiteness of $\mathcal{A}(k)$ and $\amalg(\mathcal{A} / k)$ together imply [7, Prop. 6.2.4] that $\mathcal{A}\left(\mathbb{A}_{k}\right)^{\operatorname{Br}(\mathcal{A})}$ is generated by the diagonal image of $\mathcal{A}(k)$ and the connected component of $\mathcal{A}\left(\mathbb{A}_{k}\right)$. We deduce the existence of some $Q \in \mathcal{A}(k)$ such that $\phi\left(P_{v}\right)=Q$ for all nonarchimedean primes $v$.

Given a non-archimedean prime $v$ of $k$, denote the completion of $l_{Q}$ at the prime above $v$ by $l_{Q, v}$.

\footnotetext{
${ }^{1}$ Obtainable from http://www.maths.nott.ac.uk/personal/pmxtow/maths.htm

${ }^{2}$ Obtainable from http://www.maths.nottingham.ac.uk/personal/jec/ftp/progs/
} 
From the hypotheses of the Theorem, there is some $\sigma \in \operatorname{Gal}\left(l_{Q} / k\right)$ that acts freely on $\phi^{-1}(Q)$. By the Chebotarev density theorem [2, page 227], there must be some non-archimedean prime $v$ of $k$ satisfying:

- The prime $v$ is unramified in $l_{Q}$.

- Some conjugate $\tau$ of $\sigma$ generates the Galois group of the extension $l_{Q, v} / k_{v}$.

Now as $\sigma$ acts freely on $\phi^{-1}(Q)$ then so will $\tau$. Hence there is no point in $\phi^{-1}(Q)$ that is defined over $k_{v}$. This contradicts $\phi\left(P_{v}\right)=Q$.

Proof of Theorem 1.2. Suppose that $X(k)=\emptyset$ but $X\left(\mathbb{A}_{k}\right)^{\operatorname{Br}(X)} \neq \emptyset$. Let $\left(P_{v}\right)_{v} \in X\left(\mathbb{A}_{k}\right)^{\operatorname{Br}(X)}$. As in the proof above we see that there is some $Q \in \mathcal{A}(k)$ such that $\phi\left(P_{v}\right)=Q$ for all non-archimedean primes $v$.

Choose points $R_{1}, \ldots, R_{n}$ which represent the disjoint Galois orbits of $\phi^{-1}(Q)$, and for each $i$ let $f_{i}$ be the minimal polynomial of some generator of $k\left(R_{i}\right) / k$. We note that $f_{i}$ has a root in some field $l \supseteq k$ if and only if there is an embedding $k\left(R_{i}\right) \hookrightarrow l$ fixing $k$.

Let $f=\prod f_{i}$. It follows that the polynomial $f$ has a root in some field $l \supseteq k$, if and only if there is some some point in $\phi^{-1}(Q)$ whose residue field is contained in $l$. We note that $\operatorname{deg}(f) \leq \operatorname{deg}(\phi)<6$ and $f$ has root in $k_{v}$ for all non-archimedean $v$. Hence [2, page 229] $f$ has a root in $k$, and so there is some $k$-rational point in $\phi^{-1}(Q)$. Thus $X(k) \neq \emptyset$, giving us a contradiction.

\section{Acknowledgements}

This work is funded by a grant from Sultan Qaboos University (IG/SCI/ DOMS/02/06).

I am indebted to Professor Cremona for helpful discussions, and to the referee for making valuable suggestions. 


\section{References}

[1] J.W.S. CASSEls, E.V. Flynn, Prolegomena to a middlebrow arithmetic of curves of genus 2. L.M.S. lecture notes series 230, Cambridge University Press, 1996.

[2] J.W.S. CAssels, A. Fröhlich, Algebraic number theory. Academic press, New York, 1967.

[3] D. CoraY, C. MANOIL, On large Picard groups and the Hasse Principle for curves and K3 surfaces. Acta Arith. LXXVI.2 (1996), 165-189.

[4] J.E. CREMONA, Algorithms for modular elliptic curves. second edition, Cambridge University Press, 1996.

[5] V.A. Kolyvagin, Euler systems. I The Grothendieck Festschrift, Vol. II, 435-483, Progr. Math. 87, Birkhäuser, Boston, 1990.

[6] V. ScharaschKin, The Brauer-Manin obstruction for curves. To appear.

[7] A.N. SкоRовоgatov, Torsors and rational points. Cambridge Tracts in Mathematics 144, Cambridge University Press, 2001.

Samir SIKSEK

Department of Mathematics and Statistics

Faculty of Science

Sultan Qaboos University

P.O. Box 36

Al-Khod 123, Oman

E-mail : siksek@squ.edu.om 